\title{
Plasma Window for SNS Target
}

BNL/SNS TECHNICAL NOTE

NO. 108

Deepak Raparia \& Ady Hershcovitch

April 3, 2002

COLLIDER-ACCELERATOR DEPARTMENT

BROOKHAVEN NATIONAL LABORATORY

UPTON, NEW YORK 11973 


\title{
Plasma Window for SNS Target
}

\section{Deepak Raparia and Ady Hershcovitch C-A Department, BNL, Upton, NY 11973}

\begin{abstract}
Proton beam loss between window and the target in the SNS ring to target beam transport can be completely eliminated, if Plasma Windows replace solid vacuum windows. Consequently, all problems associated with proton solid-window interaction will no longer exist. The Plasma Window is a novel apparatus, which utilized a short plasma arc to provide a vacuum-atmosphere interface without any intrusive solid structures. Extrapolating present technology to SNS implementation is a straightforward engineering issue. Among the many advantages that this technology offers are: elimination of a $4 \%$ proton beam loss, water removal from vacuum interface and elimination of radioactive water problems, as well as power and vacuum equipment savings.
\end{abstract}

\section{INTRODUCTION}

Many of the problems associated with proton beam loss in the SNS ring to target beam transport may be eliminated, if vacuum windows, which consist of two 2-mm thick inconel plates and $1.4 \mathrm{~mm}$ thick of cooling water layer in between these plates, are replaced with plasma windows. The plasma window is basically a stabilized plasma arc used as an interface between accelerator vacuum and atmosphere or pressurized targets without solid material. It has many advantages over solid windows: a plasma window (the aperture itself) can not be damaged; it can act as a lens for charged particles; it has a lower target thickness than any solid window resulting in significantly less attenuation and scattering of particles passing through it. 
Replacing the SNS ring to target beam transport window has a number of advantages among them are:

1). Elimination of a $4 \%$ proton beam loss.

2). Savings of 10's of kW, per beam line in power.

3). Water removal from vacuum interface.

4). Elimination radioactive water problems.

5). A 50-meter saving of vacuum equipment in each beam line.

This SNS Tech Note examines feasibility of a plasma window for the SNS ring to target beam transport.

\section{THE PLASMA WINDOW}

Under certain circumstances, plasmas can function as windows. A plasmas can be confined in vacuum (by electric and magnetic fields) with minimal wall contact, yet provide impedance that can balance atmospheric (or even a few atmospheres) of pressure. The use of atmospheric arc plasmas to establish a vacuum-atmosphere interface has recently been demonstrated [1]. This 'plasma window' establishes a barrier to gas flow by creating a hot plasma discharge that imparts a higher effective viscosity and a lower density than the working gas would exhibit at room temperature. Plasma windows can separate vacuum and atmosphere, or high and low vacuum, in a way that facilitates transmission of various particle beams and/or radiation from the low to high-pressure regions[2]. 
The best high-pressure results to date have been obtained using argon as both the highpressure 'target' and arc gas. In one experiment a target at 5 bar was successfully separated from atmosphere [2]. Later, a target at 2.85 bar absolute was isolated from vacuum (0.6 mbar) using a 2.36- $\mathrm{mm}$ diameter $40-\mathrm{mm}$ long arc. When coupled to a threestage differential pumping system the background pressure of $5 \times 10^{-9}$ bar was reached[3]. In a different set-up[4], these results were duplicated a 5- mm diameter 30- $\mathrm{mm}$ long arc.

Plasma windows can separate between vacuum and atmosphere (and even higher pressures), offering many advantages over presently used windows because the interaction of high-energy particles and photons with these plasmas is negligible, due to their low target thickness. A plasma window can have an effective target thickness $10^{-4}$ smaller than that of the $250 \mu \mathrm{m}$ thick Be windows often used at synchrotron light sources. In addition to fewer interactions with beams, plasma windows are impervious to thermal damage.

Transport of particles through plasma windows has also been demonstrated; a $175 \mathrm{keV}$ electron beam was transported from the vacuum into the atmosphere through the plasma window[1,2]. More recently, a $2 \mathrm{MeV}$ proton beam was successfully transmitted through a plasma window with negligible energy losses [4]. To prove their compatibility with sensitive instrumentation environments, a series of Electro-Magnetic Interference (EMI) experiments revealed that $\mathrm{rf}$ emission from the arc is negligible [2]. X-ray transmission experiments through a plasma window were performed at the National Synchrotron Light Source (NSLS) at BNL. As expected, transmission of x-rays was excellent[5]. 
Collectively these results suggest a broad range of applications including isolation windows in the Spallation Neutron Source and some of its experiments.

\section{BEAM OPTICS FOR THE PLASMA WINDOW}

The Ring to Target Beam Transport (RTBT) [6] line connects the Spallation Neutron Source (SNS)[7] accumulator ring to the target, with the required footprint of the accelerator complex. The major requirement of all parts of this accelerator is to have low uncontrolled beam losses $(<1 \mathrm{nA} / \mathrm{m})$, to allow hands on maintenance. The RTBT is equipped with two sets of halo scrapers and the ratio of aperture to the rms beam size is kept more than 3.5. Figure 1 shows the RTBT line.

The Beam requirement at the target are given in Table 1

Table1: Beam requirements at the target.

\begin{tabular}{|l|l|}
\hline Beam width & $200 \mathrm{~mm}$ \\
\hline Beam height & $70 \mathrm{~mm}$ \\
\hline $\begin{array}{l}\text { Time average current density, over beam } \\
\text { footprint }\end{array}$ & $<0.181 \mathrm{~A} / \mathrm{m}^{2}$ \\
\hline $\begin{array}{l}\text { Beam power within target and outside } \\
\text { nominal footprint }\end{array}$ & $<10 \%$ \\
\hline $\begin{array}{l}\text { Peak time-average beam current density over } \\
1 \mathrm{~cm}^{2}\end{array}$ & $<0.25 \mathrm{~A} / \mathrm{m}^{2}$ \\
\hline Peak 1-pulse density, over $1 \mathrm{~cm}^{2}$ & $\begin{array}{l}2.6 \quad \mathrm{X} \quad 10^{16} \\
\text { protons } / \mathrm{m}^{2}\end{array}$ \\
\hline
\end{tabular}




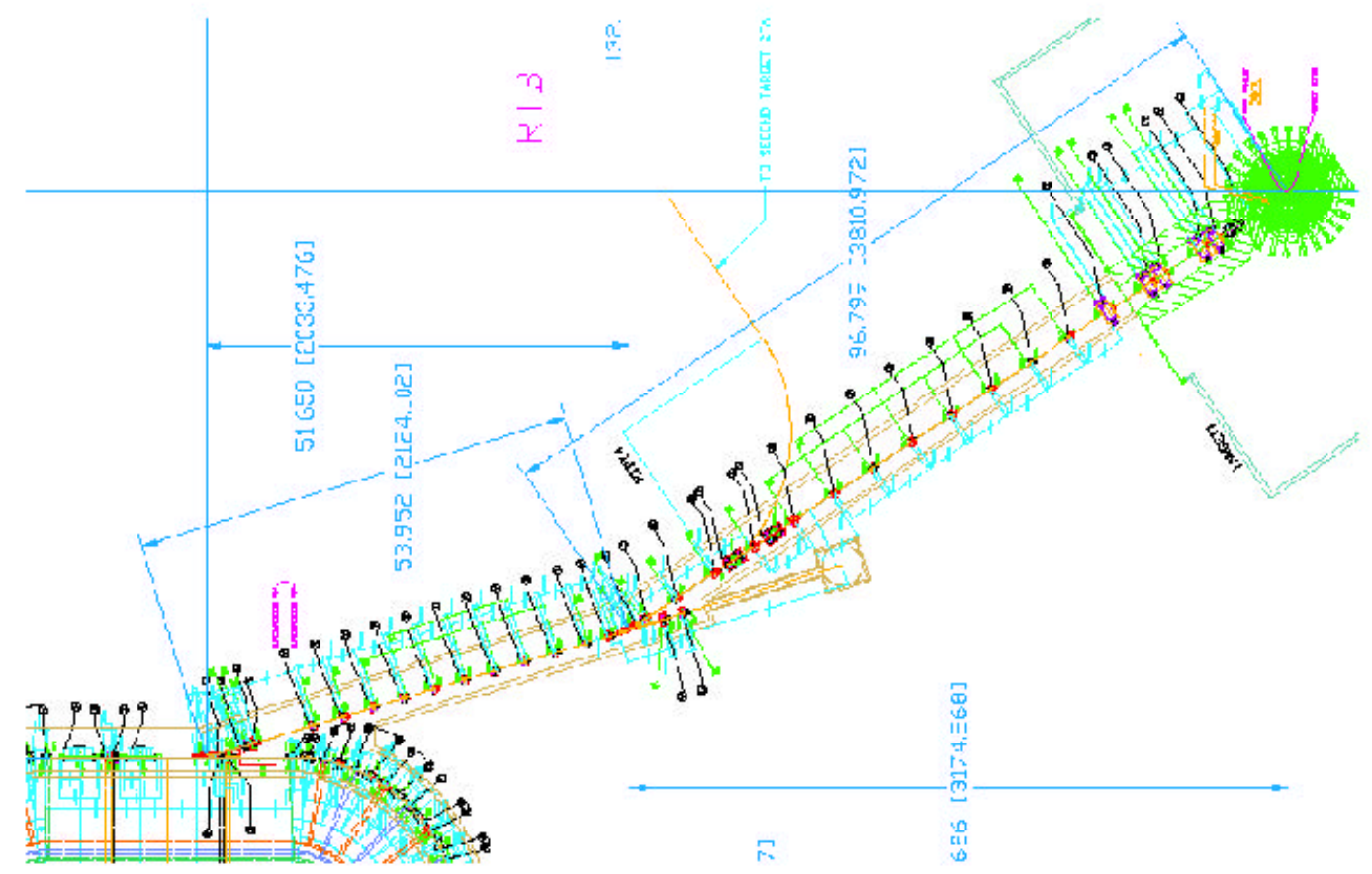

Figure 1: The layo ut of the RTBT.

The RTBT functionally can be divided into three section, (1) extraction section, (2) transport section and (3) beam spreader section. The RTBT uses a FODO Lattice up to the beam spreading section. The phase advance per cell is 90 degrees. The extraction section starts in the ring with kicker magnets and continues through six cells in the RTBT. The extraction of the beam is done in a single turn with full aperture at a pulse repetition frequency of $60 \mathrm{~Hz}$. The extraction system consists of a full aperture kicker and a Lambertson magnet septum and dipole magnet. The kick will be in vertical direction. The Lambertson septum magnet will receive the vertically kicked beam and will provide large deflection (16.8 degrees) to enable ejection horizontally from the accumulator ring. A dipole, which is 540 degrees phase advance away from the Lambertson magnet, bends 
the beam horizontally in the same direction by 16.8 degrees, making the extraction system achromatic. At end of the extraction system the horizontal dispersion and its derivative are zero. A magnet to bend the beam vertically upwards by $23.3 \mathrm{mrad}$ (opposite direction to the kicker magnet) is located about 9.3 meters away from the kicker magnet, providing a $\sim 9$ inches difference vertically between the ring and the target planes. Following the extraction system, the beam can be dumped straight through a 16.8 degrees dipole magnet. After this 16.8 degrees bend another six cells transport. The last five cell quadrupoles in the line are used for final beam spreading to produce the beam size required at the target. The beam spreader consists of five quadrupoles near the end of the RTBT. These five $32 \mathrm{~cm}$ diameters aperture quadrupoles provide the desired beam size at the target, as given in Table I. There is a $4 \mathrm{~mm}$ thick Inconel vacuum window 2.3 meters from the target. The figure 2 shows the amplitude functions $\left(\beta_{\mathrm{x}}, \beta_{\mathrm{y}}\right)$ and the dispersion function $(\eta)$ along the RTBT. 


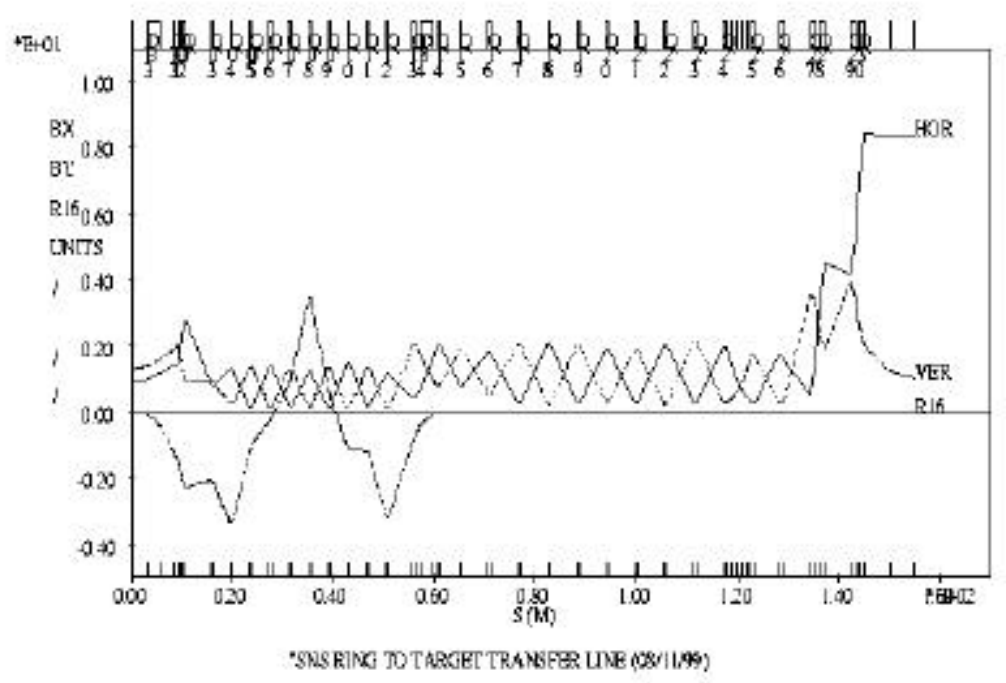

Figure 2: The amplitude functions $\left(\beta_{\mathrm{x}}, \beta_{\mathrm{y}}\right)$ and the dispersion function $(\eta)$ along the RTBT

The vacuum windows consist of two $2 \mathrm{~mm}$ thick inconel plates and $1.4 \mathrm{~mm}$ thick of cooling water layer in between these plates. The protons interact with these materials and about $4 \%$ of the proton current is lost due to nuclear scattering. To avoid these losses one can use plasma window. The one of the major requirement for the plasma window to work is smaller ( 1 inch in diameter) beam size. The figure 3 shows the low beta insertion, which can be inserted in the six cells long transport section without effecting the beam requirement at the target. The low-beta section uses the same large quadrupoles, which are used in the beam spreader section and produce the required beta 
function of 1 meter. Table II shows the required quadrupoles for this option and compares with the required quadrupoles of existing RTBT.

Table II. Required quadrupoles for low beta insertion.

\begin{tabular}{|l|l|l|}
\hline Type of quads & RTBT & RTBT with low beta insertion \\
& Number of quads & Number of quads \\
\hline $21 \mathrm{~cm}$ dia quad & 23 & 17 \\
\hline $26 \mathrm{~cm}$ dia quad & 2 & 2 \\
\hline $36 \mathrm{~cm}$ dia quad & 5 & 13 \\
\hline
\end{tabular}




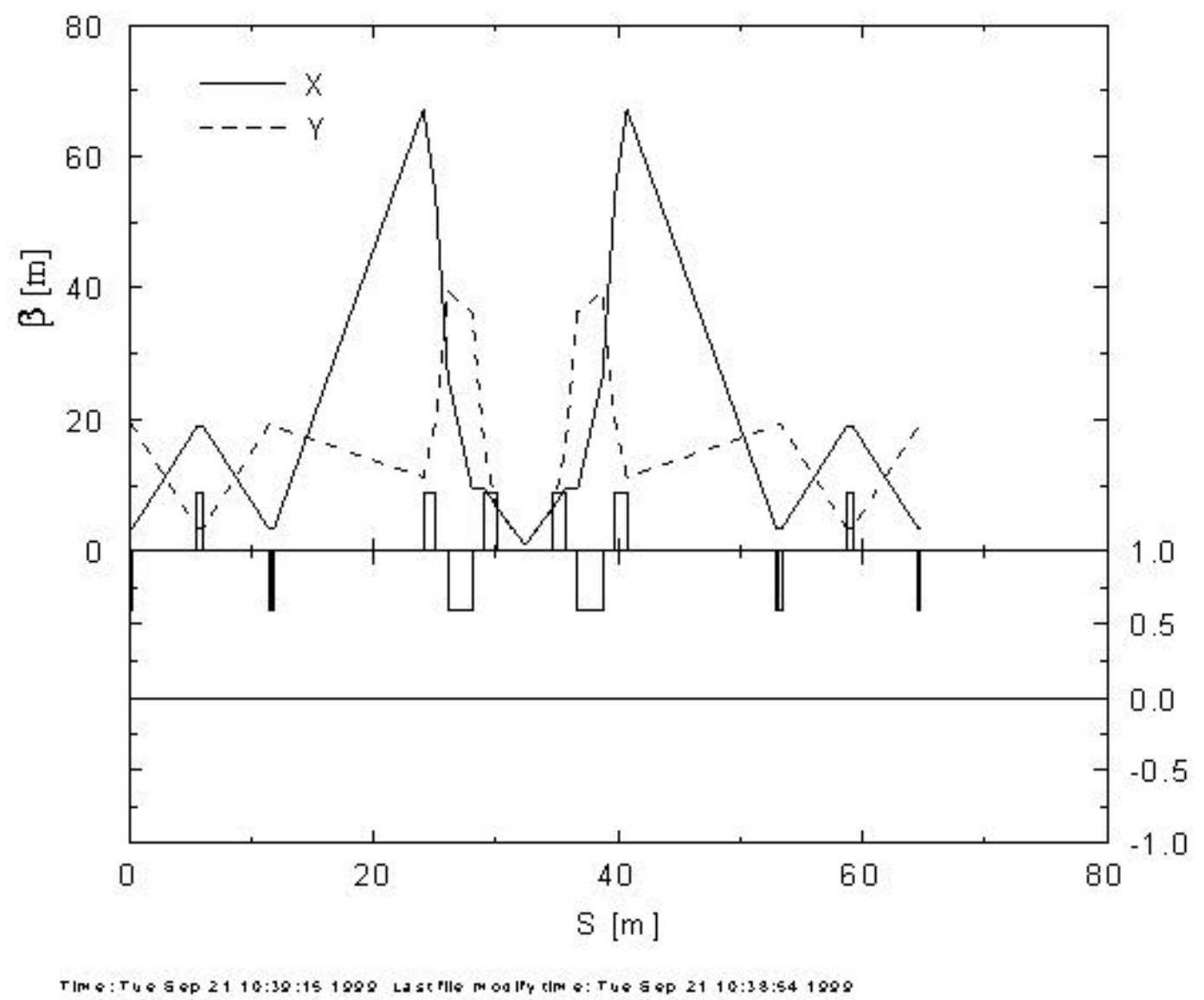

Figure 3: The low-beta section for the plasma window.

\section{A PLASMA WINDOW FOR THE RTBT LINE}

As it is shown in the previous section, the proton beam can be easily reduced to a diameter of about one inch. This beam could pass through a 2 inch diameter plasma window without any losses. Physically, the plasma window is a segmented wallstabilized plasma arc forming an interface between the high gas pressure region (atmosphere) of the target section and the vacuum (i.e., accelerator vacuum). Figure 4 is a schematic of a plasma window comprised of a wall-stabilized cascade arc discharge with a venturi. Inclusion of a venturi has shown[2] to improve vacuum separation by a factor 
of 3 , while reducing power consumption by $25 \%$. A detailed description of the plasma window can be found elsewhere[1,2].

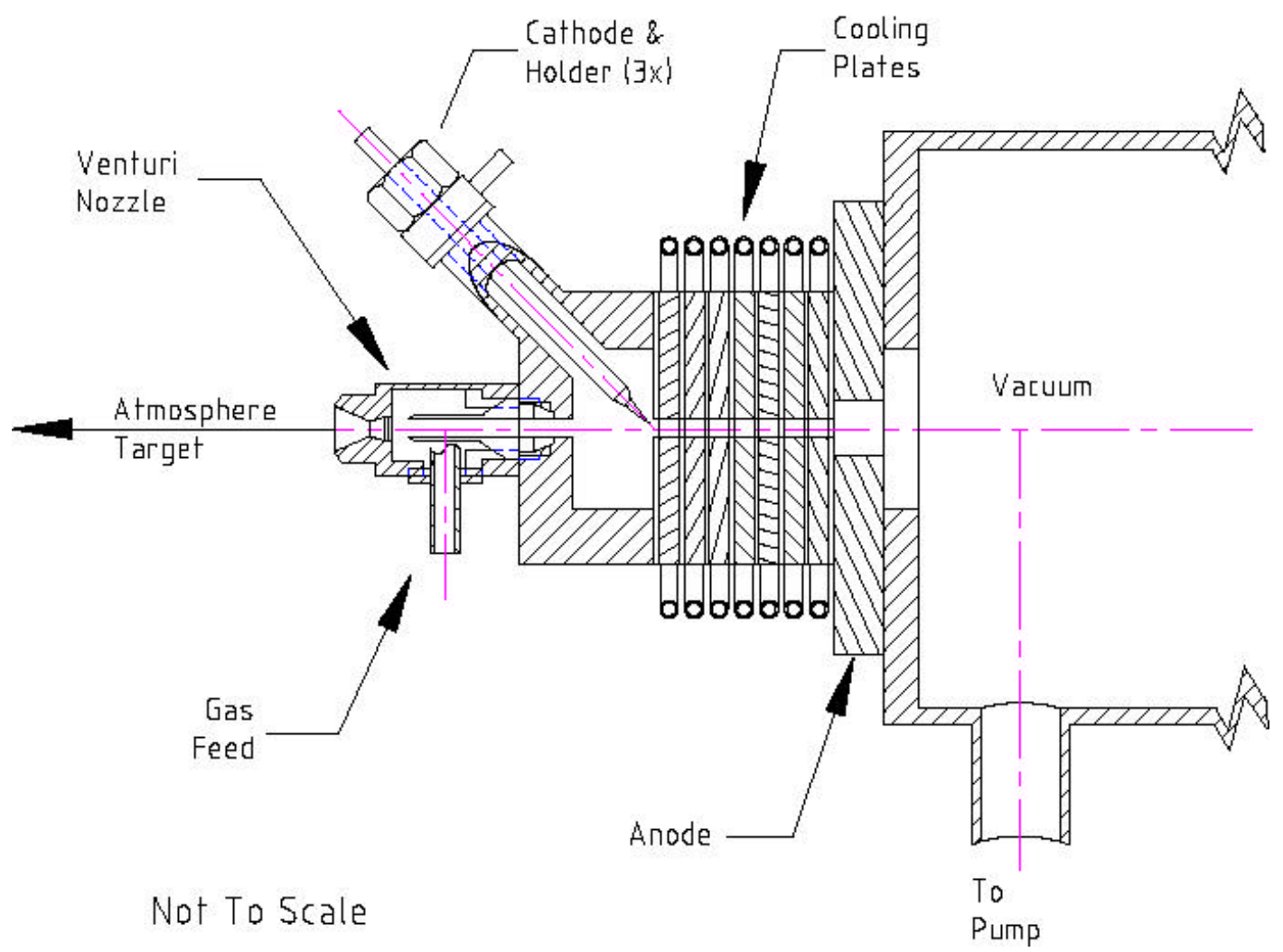

Figure 4: Schematic of a plasma window for SNS RTBT.

The arcs that have been experimented with range from $2 \mathrm{~mm}$ to $11 \mathrm{~mm}$ in diameter. Power utilized by arcs scale roughly as the arc diameter as recent experiments suggest[4]. That power scaling is roughly $10 \mathrm{~kW} / \mathrm{cm}$ of arc diameter. However, by incorporating a venturi in the arc, plasma window power is reduced by $25 \%$ [2]. With a venturi, the arc power scales as $7.5 \mathrm{~kW} / \mathrm{cm}$ of arc diameter. Thus a 2-inch arc for SNS plasma window would require a power of $38.1 \mathrm{~kW}$. This power level represents less than half of the anticipated proton beam power loss in the present SNS vacuum window. 
No physics or engineering limitation exists to prevent successful deployment of such an arc. Arcs with power levels approaching 1 MW (e.g., plasma torches) have been operating well. In principle, development of a 2-inch plasma should be a straight forward engineering problem.

\section{DISCUSSION}

Based on the preceding arguments, a plasma window in the SNS RTBT seems beneficial and feasible. It would required two additional quadrupoles (see Table II). Present SNS RTBT window design consists of two 2- $\mathrm{mm}$ thick inconel plates and $1.4 \mathrm{~mm}$ thick of cooling water layer in between these plates. The nuclear collision length is about $10 \mathrm{~cm}$ for inconel and the thickness of the inconel plate is $4 \mathrm{~mm}$. This mean probability of nuclear interaction is about $4 \%$. Simulations has shown due to nuclear scattering particle loss between window and the target is about $4 \%$ [8].

A $4 \%$ proton beam loss of a $1 \mathrm{GeV}$ beam with an average current of $2 \mathrm{~mA}$ results in an $80 \mathrm{~kW}$ loss of beam power. A 2-inch aperture plasma window will consume about half that power. The plasma window has a density that is $1 / 70$ of atmospheric density. It will have a target thickness of about $1.5 \times 10^{17} \mathrm{~cm}^{-2}$, in which the mean free path of a $1 \mathrm{GeV}$ proton is $4616 \mathrm{~cm}$, i.e., negligible attenuation is expected in a $3-6 \mathrm{~cm}$ long plasma arc. Experimental conformation was recently [4] obtained when a $2 \mathrm{MeV}$ proton beam was successfully transmitted through a plasma window with immeasurable energy and/or 
beam losses. Additionally, that plasma window had a density, which is a factor of 2.7 higher (and a correspondingly higher target thickness) than the proposed plasma window.

In addition to beam power savings, plasma window deployment will remove water from vacuum interface. Consequently, the radioactive water problems will be solved. And there will be a 50-meter saving of vacuum equipment in each beam line.

\section{Acknowledgements}

We will like to thanks W. T. Weng to suggest the problem.

\section{References:}

1. A. Hershcovitch, J. Appl. Phys. 78(9), pp. 5283-5288, (1995).

2. A. Hershcovitch, Phys. Plasmas, Vol. 5, No. 5, pp. 2130-2136, (1998).

3. W. Gerber, R. C. Lanza, A. Hershcovitch, P. Stefan, C. Castle, and E. Johnson, 'The Plasma Porthole: a Windowless Vacuum-Pressure Interface With Various Accelerator Applications', $15^{\text {th }}$ International Conference on Application of Accelerators in Research and Industry, CAARI'98, Denton, Texas, November 4-7, 1998, AIP Conference Proceedings 475.

4. A. de Beer, A. Hershcovitch, C.B. Franklyn, S. van Straaten, J. Guzek, Nuclear Instruments and Methods B Vol. 107, 259 (2000).

5. B.T. Pinkoski, I. Zacharia, A. Hershcovitch, and E. Johnson, P. Siddons, to be published in the Review of Scientific Instruments.

6. D. Raparia, J. Alessi, Y. Y. Lee and W. T. Weng, Proceeding of 1999 Particle Accelerator Conference, New York 1999, p1297 
7. B. Appleton, Proceeding of the 1997 particle accelerator conference, $p 20$

8. Franz X. Gallmeier and Deepak Raparia, to be published in the proceeding of the Fourth International Topical Meeting on Nuclear Applications of Accelerator Technology (AccApp00), Washington D.C., November 12-16, 2000. 